\title{
Mathematical model of the effect of electrodiffusion on biomineralization
}

\author{
Tianyu Zhang *, Isaac Klapper \\ Department of Mathematical Sciences, Montana State University, Bozeman, MT 59717, United States
}

\section{A R T I C L E I N F O}

Available online 23 December 2010

Keywords:

Biofilm

Calcite precipitation

Mixture model

Electrodiffusion

Electric field

\begin{abstract}
A B S T R A C T
Biofilm-induced mineral precipitation is a fundamentally important phenomenon with many potential applications including carbon sequestration and bioremediation. Based on a mixture model consisting of three phases (calcite, biofilm, and solvent) and also accounting for chemistry, mechanics, thermodynamics, fluid, and electrodiffusive transport effects, we describe the self-induced generation of an electric field due to different diffusivities of different ion species and study the effects of this field on ionic transport and calcite precipitation. Numerical simulations suggest that one of these effects is enhanced precipitation.
\end{abstract}

c 2010 Elsevier Ltd. All rights reserved.

\section{Introduction}

Biologically and chemically induced calcium carbonate formation has been proposed and/or observed to play an important role in a number of engineering, medical, and environmental contexts including carbon sequestration, co-precipitation of radionuclides (and, more generally, bioremediation in many different contexts), concrete remediation, soil stabilization, enhanced oil recovery, microbially induced corrosion, encrustation of ureteric stents, and dental caries (tooth demineralization) by dental plaques, e.g. $[2,8,14]$. Precipitation mechanics in these examples typically strongly couple biological, chemical, and physical processes through combinations of microbial dynamics and kinetics, reaction-diffusion chemistry, and fluid flow in ways that are not well understood. As a consequence, effective modeling could be useful in understanding these interactions.

In many instances, both naturally and in the lab, biomineralization occurs in combination with microbial biofilms (sessile collections of microorganisms living within a self-secreted matrix of polymers and other substances). In connection, there have been several laboratory investigations of calcite precipitation-induced by ureolytic bacteria (usually in bacterial biofilms), e.g. $[7,13,20]$. Separately, there also has been extensive work on mathematical modeling of biofilm growth and biofilm-flow interaction $[3,6,11,15]$ (including discrete, continuous and hybrid models), as well as mathematical models for solute precipitation, e.g. [21]. However, little combined modeling work has been done for biofilm-induced calcite precipitation, in part due to the complex interactions involved.

\footnotetext{
* Corresponding author.

E-mail addresses: zhang@math.montana.edu (T. Zhang), klapper@math.montana.edu (I. Klapper).
}

Recently, the authors developed a versatile mixture model which accounts for the important physical, chemical and biological processes involved in biofilm-induced calcite precipitation [23]. In this model, the system as a whole consists of three phases-biofilm, calcite and solvent-each represented by its own volume fraction. These phases satisfy conservation of mass and momentum laws with addition of a free energy of mixing. Each chemical species involved is treated as a solute in the solvent phase and represented by a concentration governed through its own advection-diffusionreaction equation. Two-dimensional numerical simulations illustrated the following phenomena: calcite precipitation occurs largely in the biofilm region, and calcite phase accumulation mainly occurs near the biofilm-solvent interface; higher ureolysis rate yields higher calcite precipitation rate; external flow can significantly change the dynamics of calcite precipitation.

The model in [23] includes electrochemistry, generally neglected in biofilm models to date (though not always, e.g. [16]). Different diffusivities of different ion species lead to charge separation through differential diffusion which in turn leads to generation of an electric field. This effect is likely to be most significant in the active layer, located along the biofilm-bulk fluid interface, where microbially induced chemistry is most active and hence where chemical gradients are particularly large. In this paper, we specifically examine the effect of the electric field on ionic transport and calcite precipitation by comparing different model outcomes with and without inclusion of electric field.

\section{Model description}

\subsection{Mechanics}

We outline, following [23], the system of equations describing the calcite precipitating system in a two-dimensional rectangular 
domain containing three phases-solvent, biomaterial, and calcite-with corresponding volume fractions denoted by $\phi_{s}, \phi_{b}$, and $\phi_{c}$ respectively. Note that $\phi_{c}+\phi_{b}+\phi_{s}=1$ so equations are needed for only two of the volume fractions. Denoting by $c$ a limiting nutrient concentration in the solvent, then the reaction-diffusion-advection equations for $c, \phi_{c}$, and $\phi_{b}$ are

$\frac{\partial}{\partial t}\left(\phi_{s} c\right)+\nabla \cdot\left(\phi_{s} c \mathbf{v}-D_{s} \phi_{s} \nabla c\right)=-\phi_{b} \frac{A c}{c_{0}+c}$

$\frac{\partial \phi_{c}}{\partial t}+\nabla \cdot\left(\phi_{c} \mathbf{v}\right)=\nabla \cdot\left(\Lambda_{c} \phi_{c} \nabla \frac{\delta f}{\delta \phi_{c}}\right)+g_{c}(S)$

$\frac{\partial \phi_{b}}{\partial t}+\nabla \cdot\left(\phi_{b} \mathbf{v}\right)=\nabla \cdot\left(\Lambda_{b} \phi_{b} \nabla \frac{\delta f}{\delta \phi_{b}}\right)+\mu \phi_{b} \frac{c}{c_{0}+c}$

where $\mathbf{v}$ is mean velocity, $p$ is pressure, and $\Lambda_{c}$ and $\Lambda_{b}$ are mobility parameters for calcite and biomaterial. The source term $g_{c}(S)$ for $\phi_{c}$ describes calcite precipitation for which we apply a commonly used quadratic rate law [24,25]

$g_{c}(S)= \begin{cases}C_{v o l} k_{p}(S-1)^{2}, & S \geq S_{\text {crit }} \\ 0, & S<S_{\text {crit }}\end{cases}$

where $k_{p}$ is the calcite precipitation rate constant and $C_{v o l}$ is the inverse molar density of mineralized calcite [12]. S, defined below, is the calcite saturation state and $S_{\text {crit }}$ is the critical supersaturation parameter.

The function $f$, the Flory-Huggins chemical free energy density of mixing [5], is given by

$$
\begin{aligned}
f\left(\phi_{c}, \phi_{b}, \phi_{s}\right)= & k_{B} T\left[\chi_{b} \phi_{b} \phi_{s}+\chi_{c} \phi_{c}\left(1-\phi_{c}\right)+\frac{\Gamma_{c}}{2}\left\|\nabla \phi_{c}\right\|^{2}\right. \\
& +\frac{\Gamma_{b}}{2}\left\|\nabla \phi_{b}\right\|^{2}+\frac{\Gamma_{s}}{2}\left\|\nabla \phi_{s}\right\|^{2} \\
& \left.+\frac{1}{N} \phi_{b} \ln \phi_{b}+\phi_{s} \ln \phi_{s}+\phi_{c} \ln \phi_{c}\right]
\end{aligned}
$$

consisting of mixing energy, transitional energy and mixing entropy components. Here $k_{B}$ is the Boltzmann constant and $T$ is temperature. The term $\chi_{c} \phi_{c}\left(1-\phi_{c}\right)$ is the calcite mixing energy density: $\chi_{c}>0$ indicates that this energy is positive for $0<\phi_{c}<1$, and that $\phi_{c}$ close to 0 or 1 is energetically preferred (favoring phase decomposition for calcite). Discussion of the other parameters in (2) as well as the extra parameters in the equations for $\phi_{b}$ and $c$ can be found in [22]. These extra parameters do not play an important role in the results presented here.

Velocity $\mathbf{v}$ and pressure $p$ are determined by modified NavierStokes equations

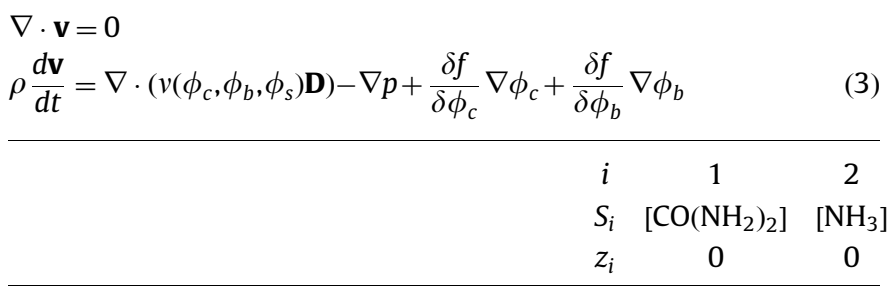

where

$v\left(\phi_{c}, \phi_{b}, \phi_{s}\right)=\phi_{c} v_{c}+\phi_{b} v_{b}+\phi_{s} v_{s}, \quad \rho=\phi_{c} \rho_{c}+\phi_{b} \rho_{b}+\phi_{s} \rho_{s}$ $\mathbf{D}=\frac{1}{2}\left(\nabla \mathbf{v}+\nabla \mathbf{v}^{T}\right)$

$v_{c}, v_{b}, v_{s}$ are viscosities of the calcite, biomaterial, and solvent, and $\rho_{c}, \rho_{b}, \rho_{s}$ are their respective densities. We approximate the solid calcite phase as an extremely viscous fluid, sufficiently viscous so that it does not deform significantly under the flow over the computation time. We do the same for the biomaterial phase. Note that for sufficiently slow flow time scales (as is the case here), biofilm can in fact be well approximated to be a viscous fluid [19]. The additional stress terms (the ones with $f$ dependence) are forces arising from the chemical free energy, see [23].

\subsection{Chemical kinetics}

We consider biomaterial containing ureolytic bacteria (e.g. Sporosarcina pasteurii). In the presence of dissolved urea, urea hydrolysis is catalyzed by such organisms to produce ammonia and carbonic acid; the subsequent ammonia-ammonium balance and carbonate species distribution lead to $\mathrm{pH}$ increase and formation of carbonate ions. In the presence of soluble calcium ions (originating from calcium chloride for example), calcite $\left(\mathrm{CaCO}_{3}\right)$ precipitation occurs once the relevant saturation state exceeds a critical level. Following again [23], we describe these kinetics based on the chemistry

$$
\begin{aligned}
& \mathrm{CO}\left(\mathrm{NH}_{2}\right)_{2}+2 \mathrm{H}_{2} \mathrm{O} \rightarrow 2 \mathrm{NH}_{3}+\mathrm{H}_{2} \mathrm{CO}_{3} \quad \text { (urea hydrolysis)-Slow } \\
& 2 \mathrm{NH}_{3}+2 \mathrm{H}_{2} \mathrm{O} \leftarrow \rightarrow 2 \mathrm{NH}_{4}^{+}+2 \mathrm{OH}^{-} \quad \text {-Fast }
\end{aligned}
$$

$\mathrm{H}_{2} \mathrm{CO}_{3}+2 \mathrm{OH}^{-} \leftarrow \rightarrow \mathrm{HCO}_{3}^{-}+\mathrm{H}_{2} \mathrm{O}+\mathrm{OH}^{-} \leftarrow \rightarrow \mathrm{CO}_{3}^{2-}+2 \mathrm{H}_{2} \mathrm{O}$-Fast

$\mathrm{CO}_{3}^{2-}+\mathrm{Ca}^{2+} \rightarrow \mathrm{CaCO}_{3} \quad$ (calcite precipitation)-Slow

Urea hydrolysis to ammonia and carbonic acid, effectively catalyzed by ureolytic bacteria [4], is described by Eq. (4). This along with calcite precipitation (Eq. (7)) are slow processes relative to diffusion. On the other hand, equilibrations (5)-(6), the two-way "fast local chemistry", together with the water self-ionization condition $\left[\mathrm{H}^{+}\right]\left[\mathrm{OH}^{-}\right]=K_{w}=10^{-14}(\mathrm{~mol} / \mathrm{L})^{2}$ occur rapidly relative to diffusion (and other processes of interest) and can thus be assumed to be at quasi-equilibrium. These fast reactions determine the ammoniaammonium balance, the carbonate species distribution, as well as the $\mathrm{pH}$. For convenience, we rewrite them in terms of $\mathrm{H}^{+}$as

$\mathrm{NH}_{3}+\mathrm{H}^{+} \stackrel{K_{3}}{\longleftrightarrow} \mathrm{NH}_{4}^{+}, \quad \mathrm{H}_{2} \mathrm{CO}_{3} \stackrel{K_{1}}{\longleftrightarrow} \mathrm{H}^{+}+\mathrm{HCO}_{3}^{-}, \quad \mathrm{HCO}_{3}^{-} \stackrel{K_{2}}{\longleftrightarrow} \mathrm{H}^{+}+\mathrm{CO}_{3}^{2-}$

Here $K_{1}, K_{2}, K_{3}$ are known equilibrium reaction rate constants under given conditions (pressure and temperature). Introduction of urea from an external source thus shifts $\mathrm{pH}$ (as a consequence of bacterially modulated ureolysis) which in turn leads to the formation of carbonate ions (Eq. (6)), which, in the presence of soluble calcium ions (supplied here by dissolved $\mathrm{CaCl}_{2}$ ), precipitate as calcite whenever the saturation state exceeds a critical supersaturation (Eq. (7)).

Chemical species introduced in (4)-(8) are described by concentrations $S_{i}$ and valences $z_{i}$, numbered as follows:

$\begin{array}{cccccccc}3 & 4 & 5 & 6 & 7 & 8 & 9 & 10 \\ {\left[\mathrm{H}_{2} \mathrm{CO}_{3}\right]} & {\left[\mathrm{NH}_{4}^{+}\right]} & {\left[\mathrm{OH}^{-}\right]} & {\left[\mathrm{HCO}_{3}^{-}\right]} & {\left[\mathrm{CO}_{3}^{2-}\right]} & {\left[\mathrm{Ca}^{2+}\right]} & {\left[\mathrm{Cl}^{-}\right]} & {\left[\mathrm{H}^{+}\right]} \\ 0 & +1 & -1 & -1 & -2 & +2 & -1 & +1\end{array}$

Species concentrations $\left(S_{i}, i \in I_{f}=\{2,3,4,5,6,7,10\}\right)$ directly participate in the fast reactions and, from (8) and the local conservation of total dissolved nitrogen $\left(N_{T}\right)$ and carbon $\left(C_{T}\right)$, pointwise satisfy

$$
\begin{aligned}
& \frac{\left[\mathrm{NH}_{4}^{+}\right]}{\left[\mathrm{NH}_{3}\right]\left[\mathrm{H}^{+}\right]}=K_{3}, \quad\left[\mathrm{NH}_{4}^{+}\right]+\left[\mathrm{NH}_{3}\right]=N_{T} \\
& \begin{aligned}
\frac{\left[\mathrm{H}^{+}\right]\left[\mathrm{HCO}_{3}^{-}\right]}{\left[\mathrm{H}_{2} \mathrm{CO}_{3}\right]} & =K_{1}, \quad \frac{\left[\mathrm{H}^{+}\right]\left[\mathrm{CO}_{3}^{2-}\right]}{\left[\mathrm{HCO}_{3}^{-}\right]} \\
& =K_{2}, \quad\left[\mathrm{H}_{2} \mathrm{CO}_{3}\right]+\left[\mathrm{HCO}_{3}^{-}\right]+\left[\mathrm{CO}_{3}^{2-}\right]=C_{T}
\end{aligned}
\end{aligned}
$$


at equilibrium. Here we assume that the activities of the chemical species can be approximated by their molar concentrations. Note that $N_{T}$ and $C_{T}$ are effectively constant with respect to equilibration of the fast reactions; they evolve only slowly according to mass transport through convection and diffusion, as well as the slow source and sink reactions (4) and (7).

By solving (9) and (10) and writing all concentrations of species involved in fast reactions in terms of $\left[\mathrm{H}^{+}\right]$, the pointwise charge neutrality constraint

$\sum_{i=1}^{10} z_{i} S_{i}=\left[\mathrm{NH}_{4}^{+}\right]+\left[\mathrm{H}^{+}\right]+2\left[\mathrm{Ca}^{2+}\right]-\left[\mathrm{OH}^{-}\right]-\left[\mathrm{HCO}_{3}^{-}\right]-2\left[\mathrm{CO}_{3}^{2-}\right]-\left[\mathrm{Cl}^{-}\right]=0$

yields the following equation for $\left[\mathrm{H}^{+}\right]$:

$$
\begin{aligned}
& \frac{N_{T} K_{3}\left[\mathrm{H}^{+}\right]}{1+K_{3}\left[\mathrm{H}^{+}\right]}+\left[\mathrm{H}^{+}\right]-\frac{K_{w}}{\left[\mathrm{H}^{+}\right]}-\frac{C_{T}}{\frac{\left[\mathrm{H}^{+}\right]}{K_{1}}+1+\frac{K_{2}}{\left[\mathrm{H}^{+}\right]}}-\frac{2 C_{T}}{\frac{\left[\mathrm{H}^{+}\right]^{2}}{K_{1} K_{2}}+\frac{\left[\mathrm{H}^{+}\right]}{K_{2}}+1} \\
& =-2\left[\mathrm{Ca}^{2+}\right]+\left[\mathrm{Cl}^{-}\right]
\end{aligned}
$$

(Note that $\left[\mathrm{Ca}^{2+}\right]$ and $\left[\mathrm{Cl}^{-}\right]$also vary only quasi-statically with respect to the fast processes.) Solving (11) pointwise in space, we then obtain $\left[\mathrm{H}^{+}\right]$and subsequently the concentration of all species involved in fast reactions.

Next we describe the effect on the species densities $S_{i}$ of the slow processes of mass transport including convection, diffusion (due to concentration gradients and to the electric field) and slow chemical reactions given by (4) and (7). We assume that the dissolved chemical species are only present in the solvent phase, and so, in its transport equation, multiply $S_{i}$ by the volume fraction of solvent $\phi_{s}$. The resulting governing equations are

$\frac{\partial\left(\phi_{s} S_{i}\right)}{\partial t}+\nabla \cdot \mathbf{J}_{i}=R_{i}, \quad 1 \leq i \leq 10$

where $\mathbf{J}_{i}$, the flux of $S_{i}$, is given by

$\mathbf{J}_{i}=\phi_{s} S_{i} \mathbf{v}-\alpha_{i} \phi_{s} S_{i} \nabla \mu_{i}$

Here $R_{i}$ is the reaction term for species $i, \alpha_{i}$ is the mobility of species $i$, and $\mu_{i}$ is the electrochemical potential of species $i$, with

$\nabla \mu_{i}=\frac{k T}{S_{i}} \nabla S_{i}+\hat{a} z_{i} \nabla \zeta$

where $\zeta$ is the electric potential (discussed below), in units of volts, and $\hat{a}$ is a units-related constant [10]. Using the Einstein relation $D_{i}=\alpha_{i} k T$ for the diffusion coefficient $D_{i}$ of species $i$, we obtain

$\frac{\partial\left(\phi_{s} S_{i}\right)}{\partial t}+\nabla \cdot\left(\phi_{s} S_{i} \mathbf{v}\right)=\nabla \cdot\left(\phi_{s} D_{i} \nabla S_{i}+a z_{i} \phi_{s} D_{i} S_{i} \nabla \zeta\right)+R_{i}$,
$\quad 1 \leq i \leq 10$

where $a=\hat{a} / k T$.

The slow process reaction functions $R_{i}$ are defined as follows. For $S_{1}$, the ureolysis reactant concentration, $R_{1}=-k_{\text {urea }} \cdot \phi_{b} \cdot S_{1}$ where $k_{\text {urea }}$ is the urea hydrolysis rate coefficient. The factor of $\phi_{b}$ is a consequence of the fact that ureolysis is catalyzed by bacteria and only occurs in the presence of biofilm. For $S_{2}=\left[\mathrm{NH}_{3}\right], S_{3}=\left[\mathrm{H}_{2} \mathrm{CO}_{3}\right]$, since they are the products of ureolysis, we have $R_{2}=-2 R_{1}$, $R_{3}=-R_{1}$ where the factor 2 in $R_{2}$ is present because one mole of urea is produced per two moles of ammonia. For $S_{4}=\left[\mathrm{NH}_{4}^{+}\right]$, $S_{5}=\left[\mathrm{OH}^{-}\right], S_{6}=\left[\mathrm{HCO}_{3}^{-}\right], S_{9}=\left[\mathrm{Cl}^{-}\right]$, and $S_{10}=\left[\mathrm{H}^{+}\right]$, we have $R_{4}=R_{5}=R_{6}=R_{9}=R_{10}=0$ since they do not participate in slow reactions. For $S_{7}=\left[\mathrm{CO}_{3}^{2-}\right], S_{8}=\left[\mathrm{Ca}^{2+}\right]$, since they are concentrations of reactants of calcite precipitation, we have

$R_{7}=R_{8}=\left\{\begin{array}{ll}-k_{p}(S-1)^{2}, & S \geq S_{\text {crit }} \\ 0, & S<S_{\text {crit }}\end{array} \quad S=\frac{\left[\mathrm{Ca}^{2+}\right]\left[\mathrm{CO}_{3}^{2-}\right]}{K_{S O}}\right.$

where $K_{S O}$ is the equilibrium calcite solubility product, $S$ is the calcite saturation state, and $k_{p}$ is the calcite precipitation rate constant. We note that the mass transport equations (12) and the mixture model equations are connected through the convection term $\nabla \cdot\left(S_{i} \mathbf{v}\right)$ and the reaction terms $R_{1}, R_{2}, R_{3}, R_{7}$, and $R_{8}$.

\subsection{Electrochemistry}

Since different ion species generally have different diffusivities (see Table 1 for example), diffusion in ionic systems leads to charge separation (pointwise charge neutrality is violated) which in turn leads to generation of an electric field. Conversely, this self-induced electric field affects ionic transport-faster diffusing ionic species tend to be "retarded" and slower ones "accelerated" [17], see below. Note that in our study, local ionic species concentrations, $\left[\mathrm{Ca}^{2+}\right]$ and $\left[\mathrm{CO}_{3}^{2-}\right]$ in particular, determine the calcite saturation state, which in turn determines calcite precipitation. Thus we include the electric field induced by spatial gradients of ions in our model. The electric field is equal to the negative gradient of the electric potential $-\nabla \zeta$, so that the second part of the flux term on the right hand side of Eq. (12) is a flux induced by the electric field. We compute the electric potential so as to enforce charge neutrality similarly to choosing fluid pressure so as to enforce divergence free velocity in the case of incompressible fluids. In particular, requiring $\sum z_{i} S_{i}=0$ (charge neutrality) and $\sum z_{i} R_{i}=0$ (charge conservation) pointwise, then multiplying Eqs. (12) by $z_{i}$ and summing over $i$ results in the following variable coefficient elliptic equation for the electric potential $\zeta$ :

$\nabla \cdot\left(\sum_{i \in I_{E}} z_{i}^{2} \phi_{s} D_{i} S_{i} \nabla \zeta\right)=-\frac{1}{a} \nabla \cdot\left(\sum_{i \in I_{E}} z_{i} \phi_{s} D_{i} \nabla S_{i}\right)$,

where $I_{E}=\{4,5,6,7,8,9,10\}$ is the index set for the charged chemical species. We note that if every chemical species has the

Table 1

\begin{tabular}{|c|c|c|}
\hline Symbol & Value & Unit \\
\hline$\chi_{c}$ & $3 \times 10^{17}$ & Dimensionless \\
\hline$v_{c}$ & $5 \times 10^{2}$ & $\mathrm{~kg} \mathrm{~m}^{-1} \mathrm{~s}^{-1}$ \\
\hline$v_{b}$ & 4 & $\mathrm{~kg} \mathrm{~m}^{-1} \mathrm{~s}^{-1}$ \\
\hline$v_{s}$ & $1.002 \times 10^{-3}$ & $\mathrm{~kg} \mathrm{~m}^{-1} \mathrm{~s}^{-1}$ \\
\hline$k_{\text {urea }}$ & 0.2 & day $^{-1}$ \\
\hline$k_{p}$ & 0.2 & $\mathrm{mmol} \mathrm{L}^{-1}$ day $^{-1}$ \\
\hline$K_{\mathrm{SO}}$ & $3.8 \times 10^{-9}$ & {$[\mathrm{~mol} / \mathrm{L}]^{2}$} \\
\hline$S_{\text {crit }}$ & 50 & Dimensionless \\
\hline$C_{v o l}$ & 32.05 & $\mathrm{~L} / \mathrm{mol}$ \\
\hline$T$ & 293.15 & Kelvin \\
\hline$a$ & 1 & $\mathrm{~kg}^{-1} \mathrm{~m}^{-2} \mathrm{~s}^{2}$ \\
\hline$\rho_{c}$ & $2.71 \times 10^{3}$ & $\mathrm{~kg} \mathrm{~m}^{-3}$ \\
\hline$\rho_{b}$ & $1 \times 10^{3}$ & $\mathrm{~kg} \mathrm{~m}^{-3}$ \\
\hline$D_{1}$ & $1.38 \times 10^{-9}$ & $\mathrm{~m}^{2} \mathrm{~s}^{-1}$ \\
\hline$D_{2}$ & $2.14 \times 10^{-9}$ & $\mathrm{~m}^{2} \mathrm{~s}^{-1}$ \\
\hline$D_{3}$ & $1.92 \times 10^{-9}$ & $\mathrm{~m}^{2} \mathrm{~s}^{-1}$ \\
\hline$D_{4}$ & $1.96 \times 10^{-9}$ & $\mathrm{~m}^{2} \mathrm{~s}^{-1}$ \\
\hline$D_{5}$ & $5.28 \times 10^{-9}$ & $\mathrm{~m}^{2} \mathrm{~s}^{-1}$ \\
\hline$D_{6}$ & $1.18 \times 10^{-9}$ & $\mathrm{~m}^{2} \mathrm{~s}^{-1}$ \\
\hline$D_{7}$ & $0.92 \times 10^{-9}$ & $\mathrm{~m}^{2} \mathrm{~s}^{-1}$ \\
\hline$D_{8}$ & $0.79 \times 10^{-9}$ & $\mathrm{~m}^{2} \mathrm{~s}^{-1}$ \\
\hline$D_{9}$ & $2.03 \times 10^{-9}$ & $\mathrm{~m}^{2} \mathrm{~s}^{-1}$ \\
\hline$D_{10}$ & $9.31 \times 10^{-9}$ & $\mathrm{~m}^{2} \mathrm{~s}^{-1}$ \\
\hline$K_{1}$ & $10^{-6.35}$ & $\mathrm{~mol} \mathrm{~L}^{-1}$ \\
\hline$K_{2}$ & $10^{-10.33}$ & $\mathrm{~mol} \mathrm{~L}^{-1}$ \\
\hline$K_{3}$ & $10^{9.25}$ & $\mathrm{~mol}^{-1} \mathrm{~L}$ \\
\hline
\end{tabular}

Parameter values used in simulations. 
same diffusion coefficient (i.e., $D_{i}$ is the same for all $i$ ), then the right hand side of (13) is zero so in this case the solution of (13) is $\zeta=$ constant, and therefore no electric field is generated. We also note that $\zeta$ depends linearly on $1 / a$ so that Eq. (12) is in fact independent of $a$.

To illustrate, consider a one-dimensional, two species example with $z_{1}=-z_{2}=1, S_{1}=S_{2}=S\left(S_{1}, S_{2}\right.$ are not the same species as defined above) and $\phi_{s}=1$, e.g., a simple dissolved salt like $\mathrm{NaCl}$. Note thus that $\nabla S_{1}=\nabla S_{2}=\nabla S$ also. For such a two species system, (13) reduces to

$\nabla \zeta=-\frac{1}{a} \frac{D_{1}-D_{2}}{D_{1}+D_{2}} \frac{\nabla S}{S}$

(in one-dimensional the constant resulting from integrating (13) is related to externally applied current, and is 0 if that current is 0 ) in which case the electrodiffusive flux for both species 1 and 2 becomes

$D_{i} \nabla S_{i}+a z_{i} D_{i} S_{i} \nabla \zeta \equiv D_{e f f} \nabla S_{i}$

where $D_{e f f}$, an effective diffusivity, is given by

$D_{\text {eff }}=\frac{2}{D_{1}^{-1}+D_{2}^{-1}}$

the harmonic average of $D_{1}$ and $D_{2}$. Thus both species diffuse at the same rate, an average weighted towards the smaller of $D_{1}$ and $D_{2}$, so that charge neutrality is automatically conserved. This effect is bounded; if, say, $D_{1} \leq D_{2}$ then $D_{1} \leq D_{\text {eff }}<2 D_{1}$ with the upper bound approached as $D_{2} \rightarrow \infty$. More generally, in the multi-dimensional, multi-species case, though the relation is not as simple, we still typically expect faster diffusing ions to be retarded and slower diffusing ones accelerated.

\section{Numerical methods}

The governing equations are solved by a finite difference method on a uniform rectangular mesh in space $(\Delta x=\Delta y=$ $1 / 256$ ). The time step size is $\Delta t=0.5 \Delta x$ which satisfies the CFL condition since the dimensionless velocity has a maximum value 1 . Computations are checked by comparison with results from doubling of the spatial resolution to ensure that the grid is adequately fine. The coupled momentum transport equation and the continuity equation are solved by a velocity-corrected projection scheme [9]. The advection-reaction-diffusion equations for quantities $\phi_{c}, \phi_{b}, c, S_{i}$ are solved by a Crank-Nicolson scheme, except that the advection term is treated by a fully implicit upwind scheme. The resulting system of algebraic equations are solved by a GMRES iterative method [18]. Computational steps are:

1. Initialize $\phi_{c}, \phi_{b}, \phi_{s}, \mathbf{v}, S_{i}, 1 \leq i \leq 10$.

2. Calculate total dissolved nitrogen $N_{T}$ and carbon $C_{T}$.

3. Solve Eq. (11) using Newton's method to obtain $\left[\mathrm{H}^{+}\right]$and $S_{i}$ for the fast equilibrating chemical species $i \in I_{f}$.

4. Solve Eq. (13) to obtain electric potential $\zeta$.

5. Time-step the transport equations (1) and momentum equations (3) to update $\phi_{c}, \phi_{b}, \mathbf{v}$.

6. Time-step the mass transport equations (12) to update slow changes in $S_{i}, 1 \leq i \leq 10$.

7. Advance time $t=t+\Delta t$ and return to step 2 .

Important parameter values used in simulations are listed in Table 1. Parameter values related to ureolysis and calcite precipitation are taken from [7], and $D_{i}, K_{1}, K_{2}, K_{3}$ are taken from [1].

We note that pointwise charge neutrality will not be enforced exactly in our numerical computations, even with electric field, due to truncation errors introduced in time-stepping equation (12). To reduce error, we modify steps 3 and 4 listed above as follows. First, when solving for $\left[\mathrm{H}^{+}\right]$after fast reaction equilibrations at time $t_{n+1}=(n+1) \Delta t$, the right hand side of Eq. (11) is corrected by adding an extra term $\sum_{i \in I_{F}} z_{i} S_{i}^{n}$ where $S_{i}^{n}$ is the concentration of species $i$ after slow changes at time $t_{n}$ (in exact arithmetic, this term would be zero). That is, the net charge is set to be the value after the slow changes at the previous time step-otherwise overall net charge would not be conserved. Second, denoting the concentration of species $i$ after fast reactions equilibrate at time $t_{n+1}$ by $\bar{S}_{i}^{n+1}$, we discretize Eqs. (12) in time the following way:

$$
\begin{aligned}
& \frac{\phi_{s}^{n+1} S_{i}^{n+1}-\phi_{s}^{n} \bar{S}_{i}^{n+1}}{\Delta t}+\nabla \cdot\left(\phi_{s}^{n+1} S_{i}^{n+1} \mathbf{v}^{n+1}\right) \\
& \quad=\nabla \cdot\left(\phi_{s}^{n} D_{i} \nabla \bar{S}_{i}^{n+1}+a z_{i} \phi_{s}^{n} D_{i} \bar{S}_{i}^{n+1} \nabla \zeta^{n+1}\right)+R_{i}^{n+1}, \quad 1 \leq i \leq 10
\end{aligned}
$$

Note that convection and reaction terms are treated implicitly, while the other flux terms are treated explicitly. Recall again that $\sum_{i \in I_{E}} z_{i} \bar{S}_{i}^{n+1} \neq 0$ under truncated arithmetic. In order then to more nearly enforce exact charge neutrality again at time $t_{n+1}$, we set the implicit constraint $\sum_{i \in I_{E}} z_{i} S_{i}^{n+1}=0$ (which also implies $\sum_{i \in I_{E}} z_{i} R_{i}^{n+1}=0$ ). Then, multiplying Eqs. (14) by $z_{i}$ and summing over $i$ results in the following discretization of the equation for the electric potential $\zeta^{n+1}$ at time $t_{n+1}$ :

$$
\begin{aligned}
\nabla & \cdot\left(\sum_{i \in I_{E}} z_{i}^{2} \phi_{s}^{n} D_{i} \bar{S}_{i}^{n+1} \nabla \zeta^{n+1}\right) \\
= & -\frac{1}{a}\left[\nabla \cdot\left(\sum_{i \in I_{E}} z_{i} \phi_{s}^{n} D_{i} \nabla \bar{S}_{i}^{n+1}\right)+\sum_{i \in I_{E}} \frac{\phi_{s}^{n} z_{i} \bar{S}_{i}^{n+1}}{\Delta t}\right]
\end{aligned}
$$

This potential describes an electric field which in fact acts to reduce numerically introduced charge non-neutrality.

\section{Results and discussion}

\subsection{Biomineralization without external flow}

We consider a two-dimensional system with a square domain of size $1 \mathrm{~mm}$ by $1 \mathrm{~mm}$. Initial conditions consist of two semicircular biofilm colonies (containing ureolytic bacteria) attached to the top and bottom boundaries as well as uniform concentrations of dissolved urea and calcium chloride with concentrations 6 and $2 \mathrm{mmol} / \mathrm{L}$ respectively. The system is initially $\mathrm{pH}$ neutral and contains no calcite. Initial values of the chemical species (in units of $\mathrm{mol} / \mathrm{L})$ are $\left[\mathrm{CO}\left(\mathrm{NH}_{2}\right)_{2}\right]=6 \times 10^{-3},\left[\mathrm{Ca}^{2+}\right]=2 \times 10^{-3}$, $\left[\mathrm{Cl}^{-}\right]=4 \times 10^{-3},\left[\mathrm{NH}_{3}\right]=\left[\mathrm{H}_{2} \mathrm{CO}_{3}\right]=\left[\mathrm{NH}_{4}^{+}\right]=\left[\mathrm{HCO}_{3}^{-}\right]=\left[\mathrm{CO}_{3}^{2-}\right]=0$, $\left[\mathrm{H}^{+}\right]=\left[\mathrm{OH}^{-}\right]=10^{-7}$ (neutral $\mathrm{pH}$ ). We set $c=0$ and thus allow no growth of biomaterial. For the moment, we set the velocity to be zero at the domain boundary, e.g. no external flow. In this case with no external flow, no-flux boundary conditions are used for Eq. (12). Fig. 1(a) shows the initial contours of $\phi_{b}$ (biomaterial volume fraction). There is little change in $\phi_{b}$ during the simulation since the biofilm does not grow and mechanical deformation from fluid flow is small in the absence of externally driven flow. As described previously, in the presence of soluble calcium ions, microbially catalyzed urea hydrolysis eventually leads to calcite precipitation. We run the simulation with the electric field and then without it (by omitting step 4 in the numerical method and the term containing $\zeta$ in Eq. (12)), and compare the results to demonstrate the electrochemical effect on ionic transport and calcite precipitation. In all cases, we run for a time period of 1 day (approximately the time period of a typical experimental system and a long enough time to see significant precipitation). 

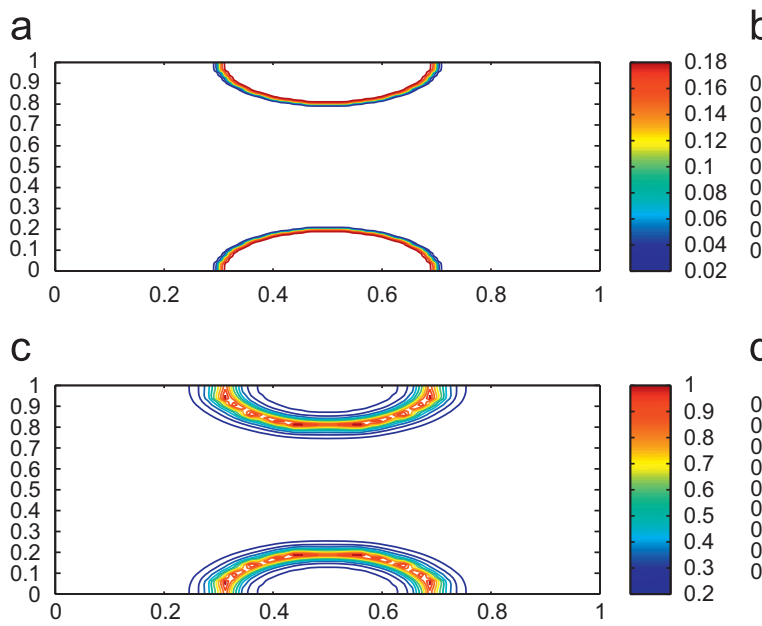

b

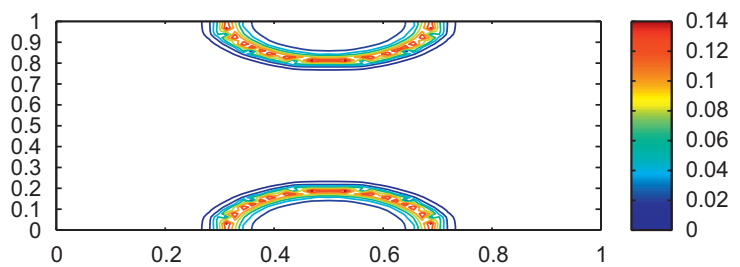

d

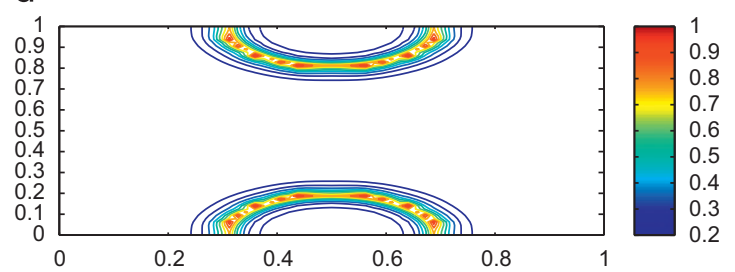

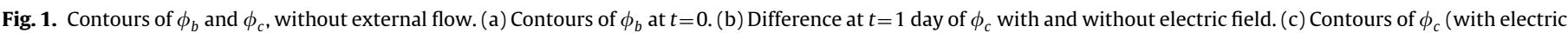

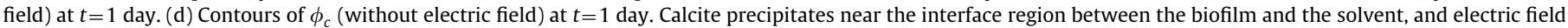
enhances calcite precipitation with largest difference approximately 0.15 .

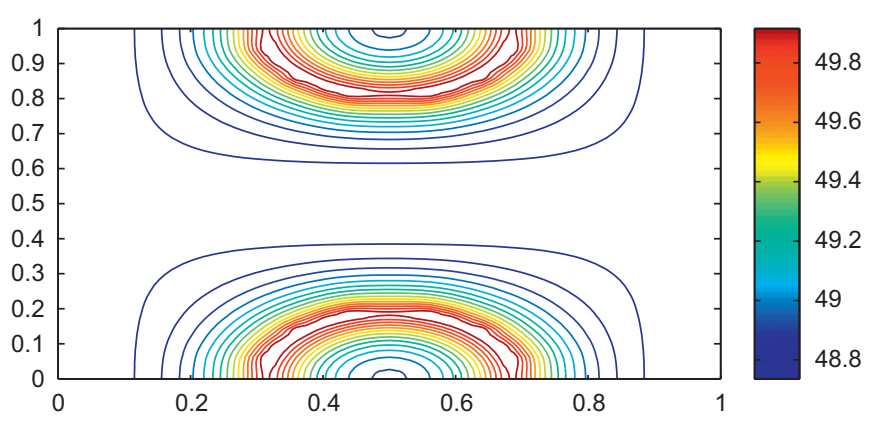

Fig. 2. Contours of calcite saturation state $S$ at $t=1$ day, without external flow, with electric field.

Fig. 1(c) shows contours of $\phi_{c}$ (calcite volume fraction) at $t=1$ day with electric field; Fig. 1 (d) shows them without electric field. We observe that, in both cases, calcite precipitation mainly occurs in the interface region between the biofilm and the solvent, and that it nearly forms a shell of calcite surrounding the biofilm. The biofilm-solvent interface allows access to both bacteria catalyzing ureolysis as well as urea and calcium ions from the surrounding bulk fluid. Thus the calcite saturation state $S$ is large there, leading to calcite precipitation, see Fig. 2.

Fig. 1(b) shows contours of the difference between $\phi_{c}$ given in (c) and (d), i.e., with and without electric field, illustrating that the volume fraction $\phi_{c}$ calculated with electric field is generally larger than that calculated without electric field. The largest difference in volume fraction is approximately 0.15 (i.e., $15 \%$ ), indicating that electric field does have a significant effect on calcite precipitation. Below we will discuss the effect in detail.

Fig. 3(a) shows relative net charge concentration of the system $\Sigma_{i} z_{i} S_{i} / \Sigma_{i}\left|z_{i}\right| S_{i}$ at $t=1$ day including electric field, while Fig. 3(b) shows relative net charge concentration computed without electric field. Note that the error shown in (a) is an order of magnitude smaller, which indicates that including the electric field does help enforce charge neutrality as we expect (charge neutrality is not enforced exactly due to truncation error of the numerical scheme).

Fig. 4(a) shows the calcium concentration $\left[\mathrm{Ca}^{2+}\right]$ at $t=1$ day computed with electric field and 4(b) shows the same except computed without electric field. Note that they have similar shape. However, the difference between $\left[\mathrm{Ca}^{2+}\right]$ given in (a) and (b) is shown in Fig. 4(c) and we observe that $\left[\mathrm{Ca}^{2+}\right]$ calculated with electric field is larger in the biofilm-occupied region and smaller in the bulk fluid region. This can be explained by observing the electric potential $\zeta$ at $t=1$ day, see Fig. 4(d). Since the electric field is given by $-\nabla \zeta$ which points from the bulk fluid region towards the biofilm region near the biofilm-solvent interface, and $\mathrm{Ca}^{2+}$ is a positive ion which tends to move along the electric field, we observe that the electric field enhances diffusion of $\mathrm{Ca}^{2+}$ from the bulk fluid to the biofilm-occupied region where it subsequently can be precipitated. We note that $\mathrm{Ca}^{2+}$ diffusion is "accelerated" because it has the smallest diffusion coefficient $\left(D_{8}=0.79 \times 10^{-9}\right.$ in Table 1).

Fig. 5 shows contours of the diffusive flux of $\left[\mathrm{Ca}^{2+}\right]=S_{8}$ at $t=1$ day computed with electric field; (a), (b) shows the $x$ - and $y$ components of the passive component $-\phi_{s} D_{8} \nabla S_{8}$ of diffusive flux, and (c), (d) shows the $x$ - and $y$-components of the electrodiffusive component $-a z_{8} \phi_{s} D_{8} S_{8} \nabla \zeta$. Observe that these two flux components have similar shape, and both diffuse calcium ions from the bulk fluid region towards the biofilm-occupied region where calcite is precipitated. Thus the electric field has the effect of accelerating diffusion of the slow diffusing calcium ions. Note also that the peak value of the electrodiffusive component is about $40 \%$ of that of the passive component, indicating its significance. In contrast, Fig. 6 shows contours of the passive diffusive flux $-\phi_{s} D_{8} \nabla S_{8}$ of calcium ions at $t=1$ day computed without electric field; (a), (b) shows the $x$ - and $y$-components respectively. The flux has a similar shape as those shown in Figs. 5(a) and (b), with a peak value about 1.5 times larger than before. This indicates that, without electric field, there is a sharper gradient of $\left[\mathrm{Ca}^{2+}\right]$ between the bulk fluid region and the biofilm-occupied region at $t=1$ day, i.e., transport of calcium ion is less efficient as compared to the case with electric field (thus leading to sharper gradients).

\subsection{Biomineralization with external flow}

We now consider the same system except with an externally driven flow (parabolic inflow with maximum velocity $0.1 \mathrm{~mm} / \mathrm{s}$ ); Reynolds number is approximately 1 and Peclet number is approximately 100 . Dissolved urea and calcium chloride (concentrations 6 and $2 \mathrm{mmol} / \mathrm{L}$ respectively) enter the domain with the influent from the left boundary; effluent leaves the domain from the right boundary. The velocity at the top and bottom boundaries is zero. Fig. 7(a) shows contours of $\phi_{b}$ at $t=1$ day computed with electric field. We observe that, due to the external flow, biofilm is sheared in the downstream direction and elongated. Contours of $\phi_{b}$ 
a

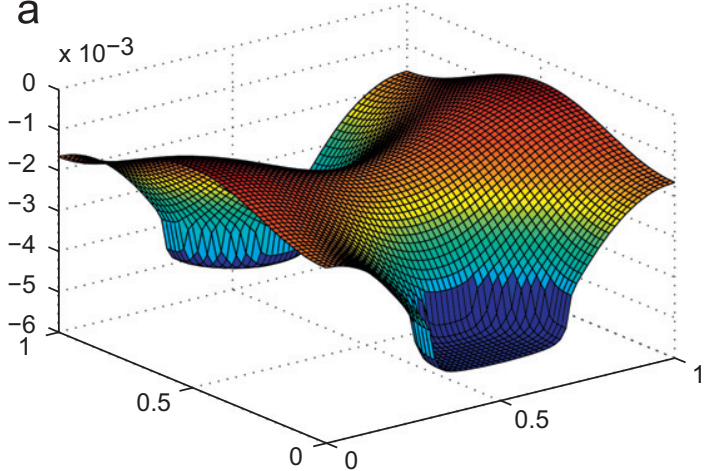

b

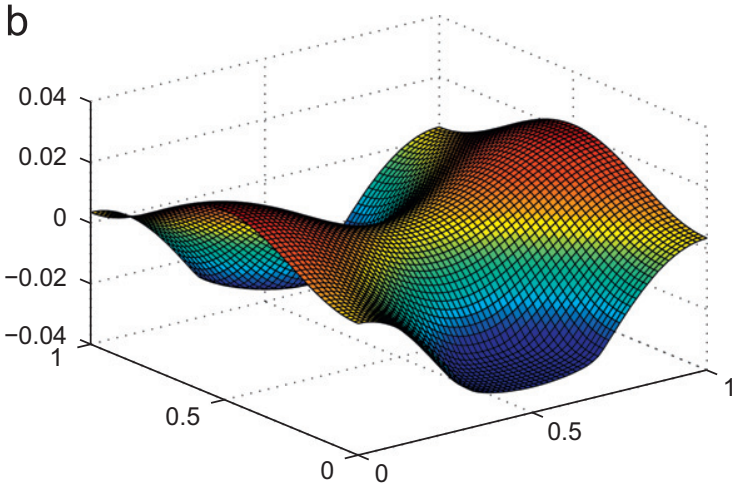

Fig. 3. Profiles of relative net charge concentration at $t=1$ day without external flow (a) with electric field and (b) without electric field.

a

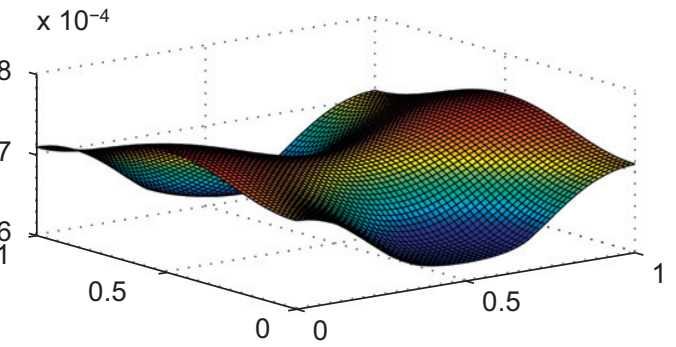

C

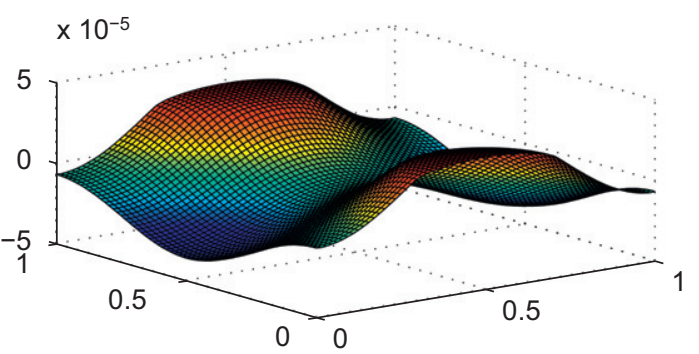

b

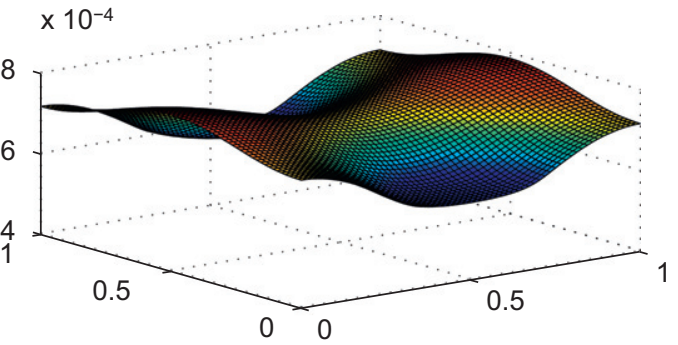

d

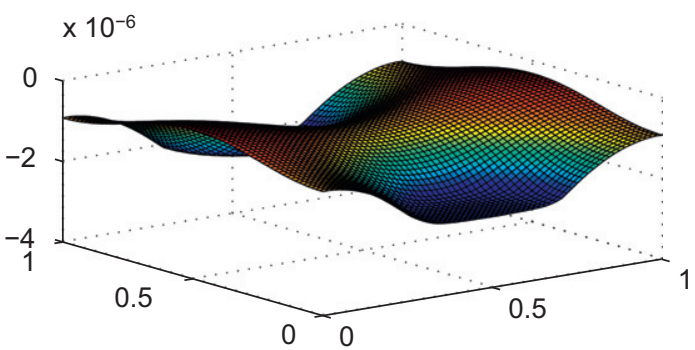

Fig. 4. Profiles of $\left[\mathrm{Ca}^{2+}\right]$ and electric potential $\zeta$, without external flow. (a) $\left[\mathrm{Ca}^{2+}\right]$ concentration at $t=1$ day with electric field. (b) $\left[\mathrm{Ca}^{2+}\right]$ concentration at $t=1$ day without electric field. (c) Difference of (a) and (b). (d) Electric potential $\zeta$ at $t=1$ day. Electric field enhances the diffusion of $\mathrm{Ca}^{2+}$ from the bulk fluid to the biofilm-occupied region.
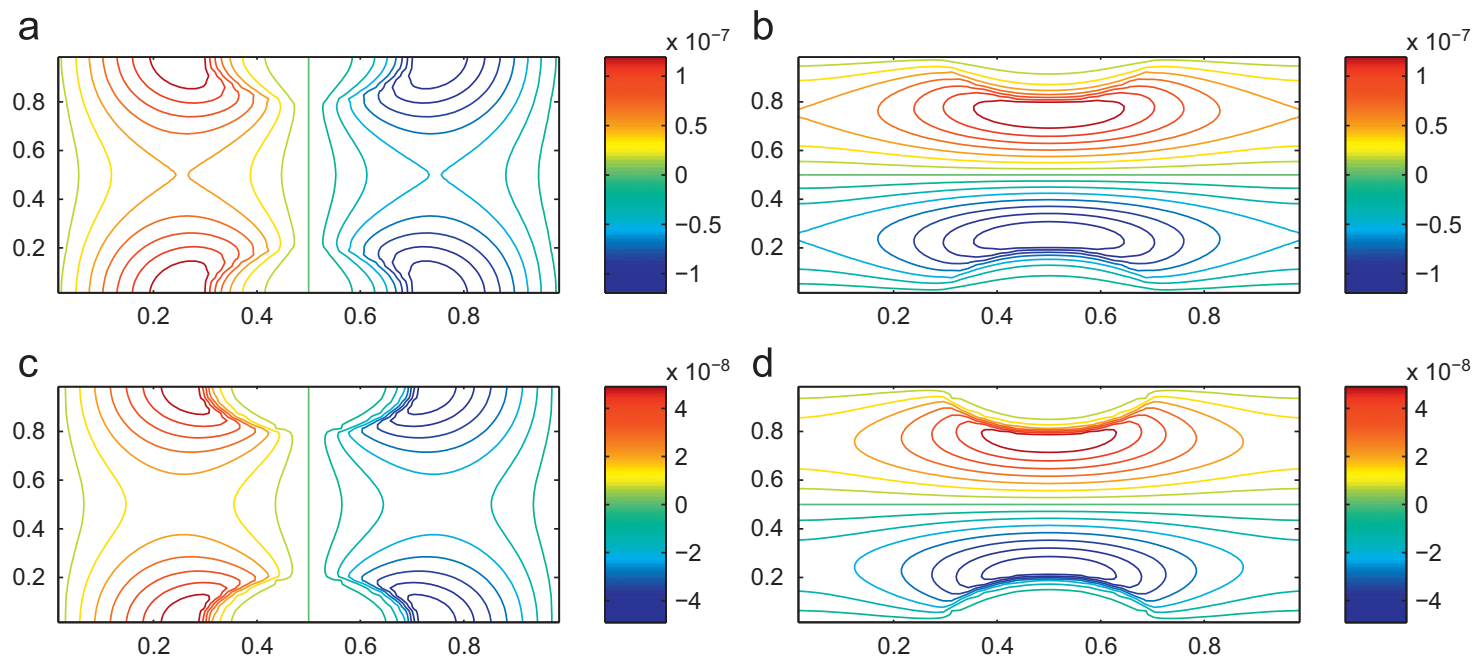

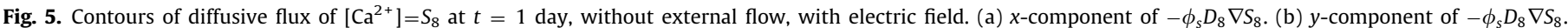
(c) $x$-component of $-a z_{8} \phi_{s} D_{8} S_{8} \nabla \zeta$. (d) $y$-component of $-a z_{8} \phi_{s} D_{8} S_{8} \nabla \zeta$. 
calculated without electric field are similar to those shown in Fig. 7(a) and are omitted. Figs. 7(c) and (d) show contours of $\phi_{c}$ at $t=1$ day calculated with and without electric field respectively. We again observe in both cases that calcite phase accumulation mainly occurs along the biofilm-bulk fluid interface. Fig. 7(b) shows contours of the difference between $\phi_{c}$ as given in (c) and (d). As without external flow, $\phi_{c}$ calculated with electric field is generally larger than $\phi_{b}$ calculated without; the largest difference now, though, is smaller at approximately 0.08 (i.e., $8 \%$ ). We remark, by the way, that the shape of the calcite contours shown in Figs. 7(b)-(d) is not directly due to elongation caused by shear stress of the flow. Rather, shear deforms the biofilm and resulting localized chemical activity (ureolysis and calcite precipitation) around the deformed biofilmbulk fluid interface produces calcite.

Figs. 8(a) and (b) show the net relative charge density of the system $\Sigma_{i} z_{i} S_{i} / \Sigma_{i}\left|z_{i}\right| S_{i}$ at $t=1$ day calculated with and without electric field respectively; as before the result with field is an order of magnitude smaller than without indicating that including the electric field does enforce charge neutrality.

By plotting $\left[\mathrm{Ca}^{2+}\right]$, we can again conclude that the electric field enhances the diffusion of $\mathrm{Ca}^{2+}$ and eventually leading to more calcite precipitation, figures not shown. On the other hand, we
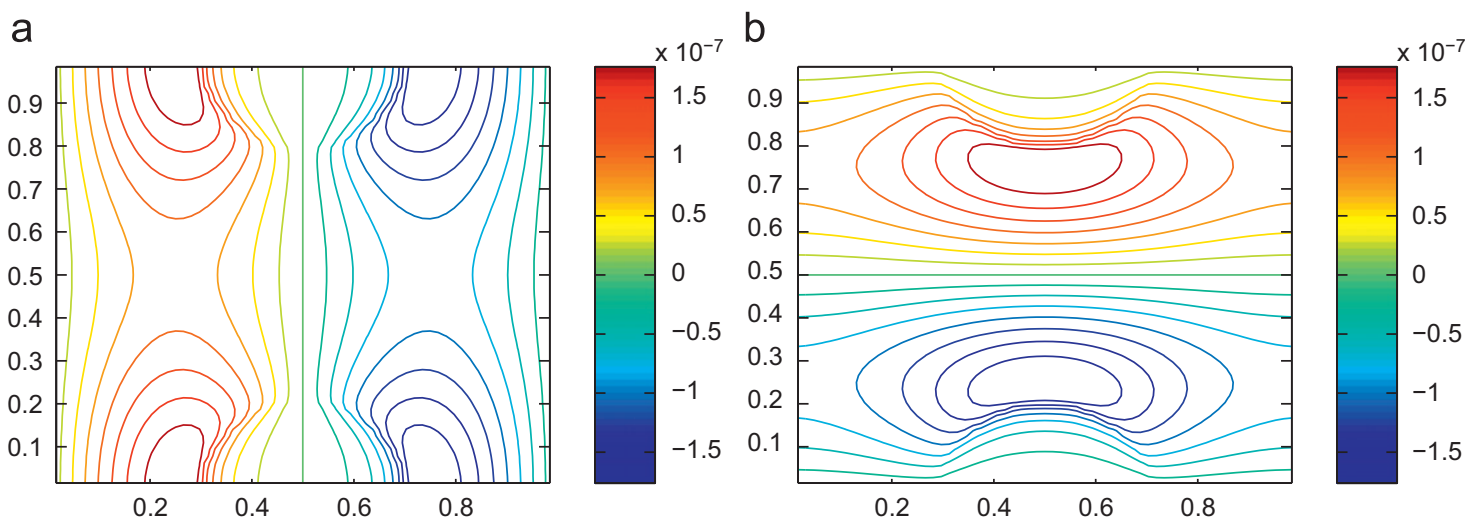

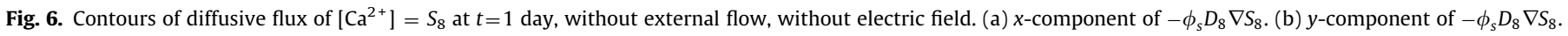

a

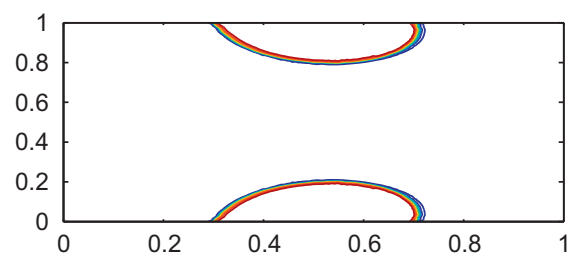

C

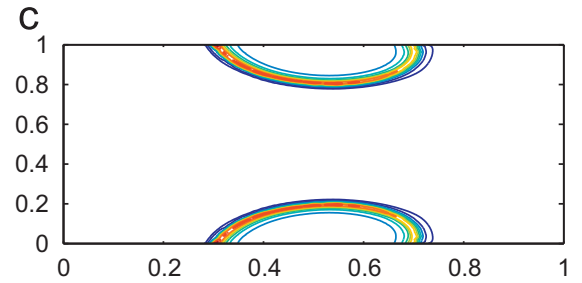

b
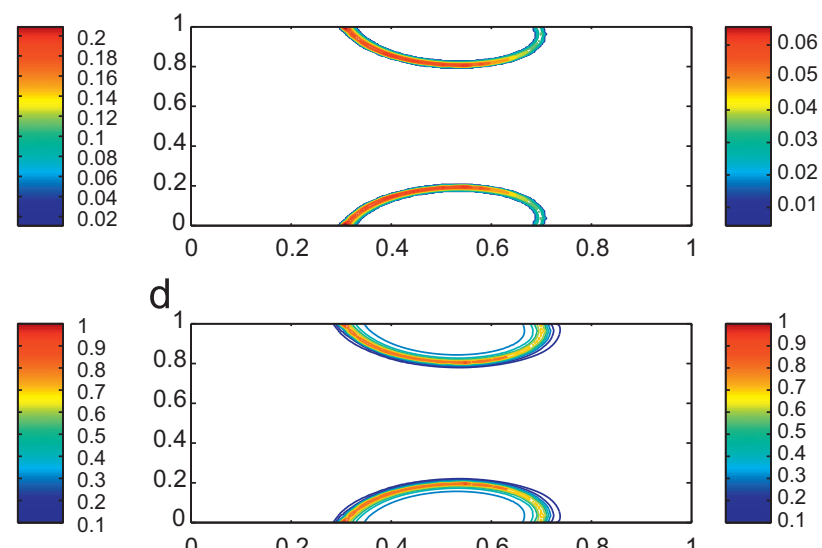

d

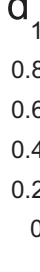

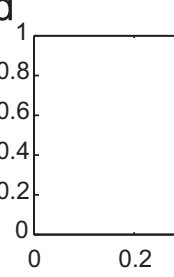
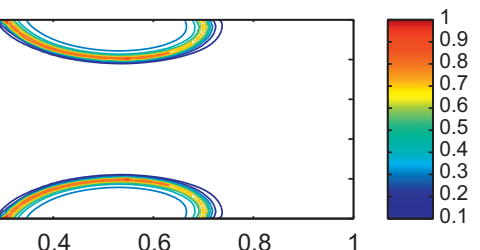

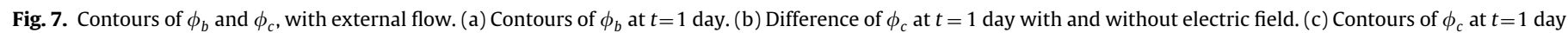

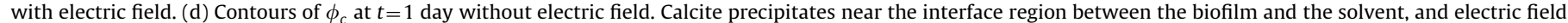
enhances calcite precipitation with biggest difference approximately 0.08 .

a

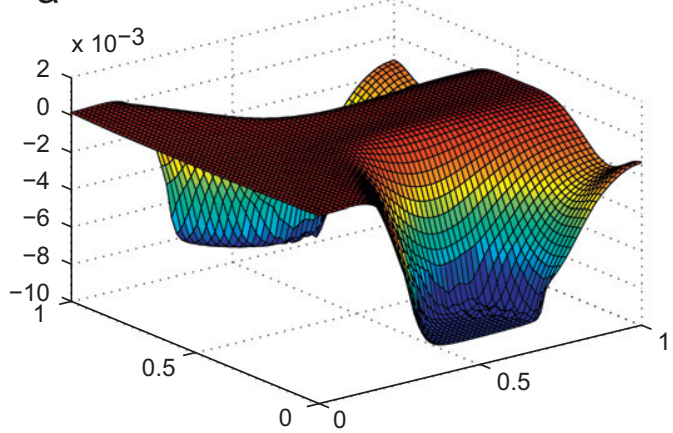

b

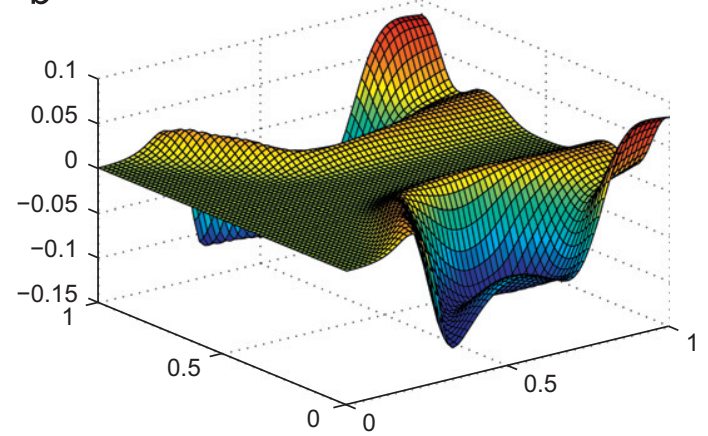

Fig. 8. Profile of relative net charge density at $t=1$ day, with external flow (a) with electric field and (b) without electric field. 
a

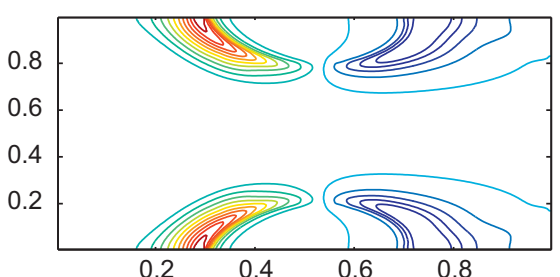

C

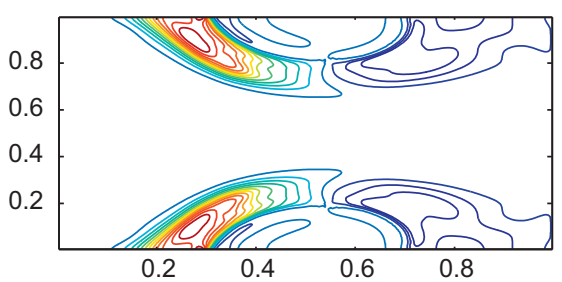

b
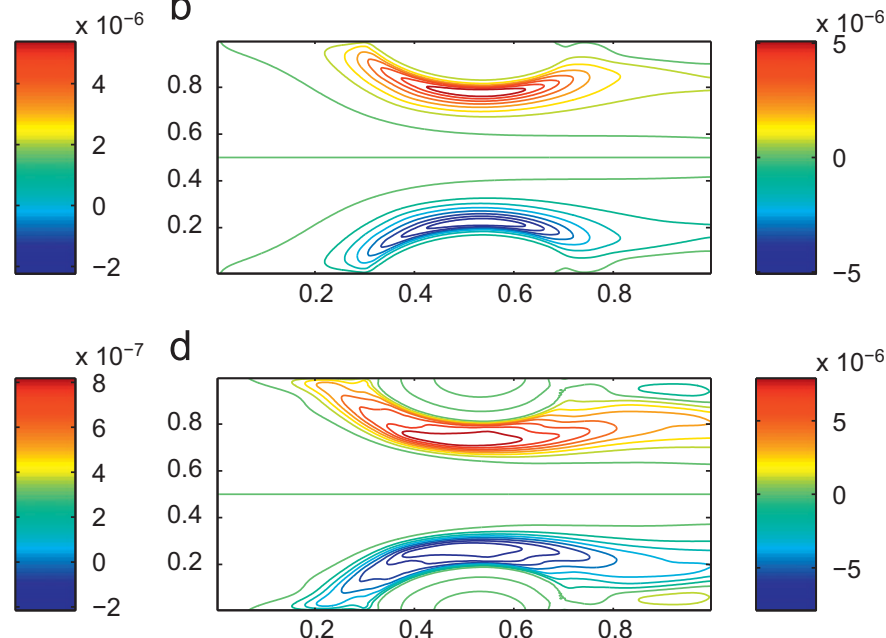

d

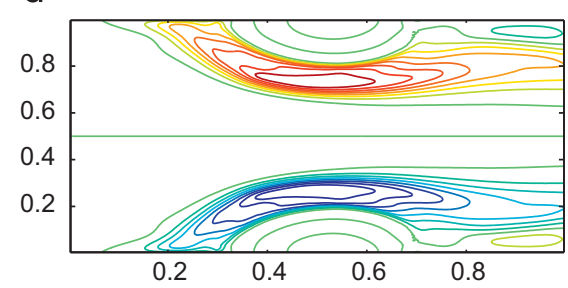

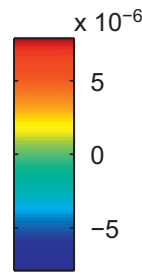

Fig. 9. Contours of components of diffusive flux of $\left[\mathrm{Ca}^{2+}\right]=S_{8}$ at $t=1$ day, with external flow, with electric field. (a) $x$-component of $-\phi_{s} D_{8} \nabla S_{8}$. (b) $y$-component of $-\phi_{s} D_{8} \nabla S_{8}$. (c) $x$-component of $-a z_{8} \phi_{s} D_{8} S_{8} \nabla \zeta$. (d) $y$-component of $-a z_{8} \phi_{s} D_{8} S_{8} \nabla \zeta$.

a

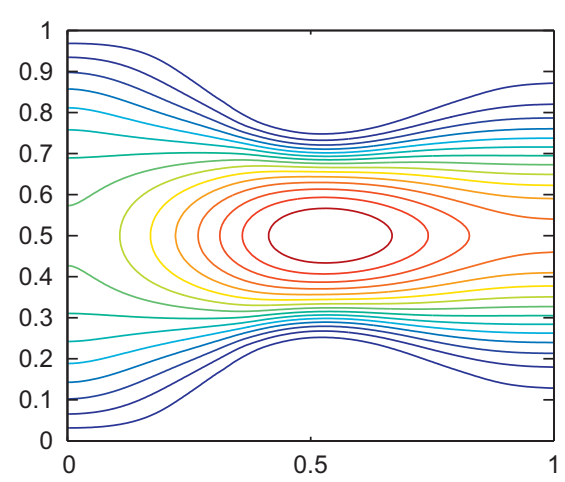

b

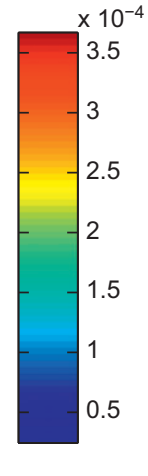

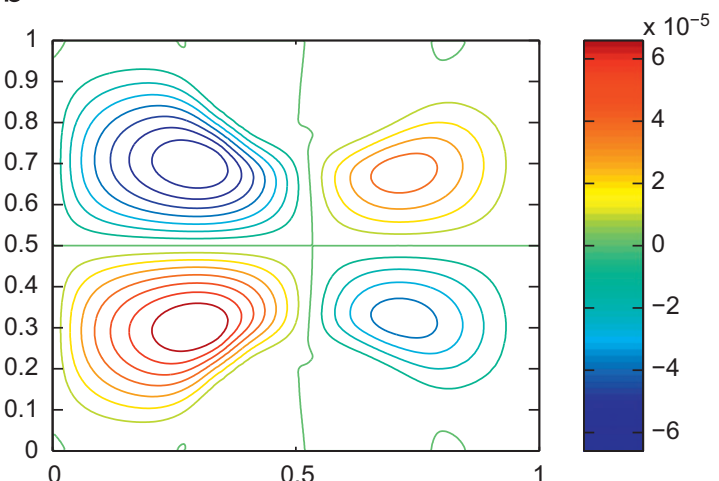

Fig. 10. Contours of advective flux of $\left[\mathrm{Ca}^{2+}\right]=S_{8}$ at $t=1$ day, with external flow, with electric field. (a) $x$-component of $\phi_{s} \mathbf{v} S_{8}$. (b) $y$-component of $\phi_{s} \mathbf{v} S_{8}$.

observe that the difference between $\phi_{c}$ values calculated with and without $\zeta$ is smaller in the presence of external flow (approximately 0.08 in this case), while it is approximately 0.15 when there is no external flow. In the presence of external flow, advection serves to transport mass, lessening the importance of diffusion and hence of electric field.

Fig. 9 shows the contours of the diffusive flux of $\left[\mathrm{Ca}^{2+}\right]=S_{8}$ at $t=1$ day computed with electric field and external flow; (a), (b) shows the $x$ - and $y$-components of the passive diffusive flux $-\phi_{s} D_{8} \nabla S_{8}$, and (c), (d) shows the $x$ - and $y$-components of the electrodiffusive flux $-a z_{8} \phi_{s} D_{8} S_{8} \nabla \zeta$. We again observe that these two flux types have similar shape, and both transport calcium ion from the bulk fluid region towards the biofilm-occupied region where it is consumed. In contrast to the no-flow case, as a consequence of advective transport the contours lines are stretched in the downstream direction, and the $x$-component of the flux has a larger value to the left of the biofilm-occupied region than to the right (since the flow carrying calcium ions goes from left to right). In the bulk fluid region $\left[\mathrm{Ca}^{2+}\right]$ is nearly constant (concentration from the influent) and its gradient is negligible; thus diffusive transport mainly occurs near the solvent-biofilm interface and forms a diffusion layer there. The peak value of $-\phi_{s} D_{8} \nabla S_{8}$ is about six times larger than the peak value of $-a z_{8} \phi_{s} D_{8} S_{8} \nabla \zeta$, suggesting that the contribution to the flux by the electric field is less important than in the case without flow. The diffusive flux contours computed with external flow but without electric field have similar shapes as those shown in Figs. 9(a) and (b), but with a peak value about 1.2 times larger than before, figure not shown.

To further illustrate the effect of the external flow, Fig. 10 shows contours of the advective flux $\phi_{s} \mathbf{v} S_{8}$ of $\left[\mathrm{Ca}^{2+}\right]$ at $t=1$ day computed with electric field and external flow; (a), (b) show the $x$ - and $y$-components respectively. We observe that the advective transport mainly occurs in the bulk fluid region, and its magnitude decreases close to the solvent-biofilm interface. For the $x$-component, the peak value of the advective flux is about 66 times larger than that of the diffusive flux, and for the $y$-component, the peak value of the advective flux is about 13 times larger than that of the diffusive flux. Thus advection is the main mechanism for ion species transport in the bulk fluid region, and diffusion becomes important only in the diffusion layer near solvent-biofilm interface.

\section{Conclusion}

To summarize, electric field serves to accelerate the transport of the slowest diffusing ion species, namely in this case calcium ion; the effect is most prominent when diffusion is most dominant over advection. The significance of this acceleration is that transport of the slowest diffusing ionic species may often be the rate limiting bottleneck and hence electric field acceleration may be able to increase reaction rates in such instances. We illustrate the principle by varying the calcium ion diffusivity (this of course can be easily done computationally but not in 


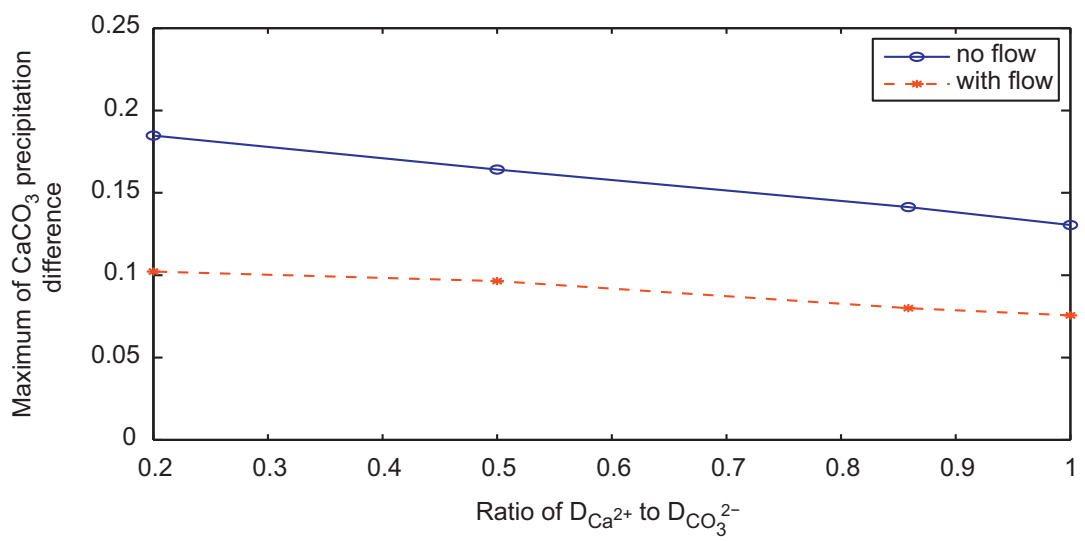

Fig. 11. Largest difference between $\phi_{c}$ calculated with and without the electric field, plotted against the ratio of calcium and carbonate ion diffusivities.

reality). That is, the computations presented above were repeated with the same ion diffusivities as given in Table 1 except that the calcium ion diffusivity was varied. In particular, $D_{\mathrm{CO}_{3-}^{2-}}=D_{7}=0.92 \times$ $10^{-9}, D_{\mathrm{Ca}^{2+}}=D_{8}=0.79 \times 10^{-9}$ but to illustrate the importance of electric field acceleration of transport of the slowest diffusing ion species, we repeated the computation using different diffusivity ratios $D_{\mathrm{Ca}^{2+}} / D_{\mathrm{CO}_{3}^{2-}}=1,0.5,0.2$ (the true ratio is around 0.86 ), where the same $D_{\mathrm{CO}_{3}^{2-}}$ is used but $D_{\mathrm{Ca}^{2+}}$ is changed to achieve the given ratio and all other parameters are left unchanged. (The significance of $\mathrm{CO}_{3}^{2-}$ is that it is the co-reactant with calcium ion in calcite precipitation-hence precipitation requires transport of both species to the biofilm-bulk fluid interface, and thus choosing $D_{\mathrm{CO}_{3}^{2-}}$ for normalization seems natural.)

Fig. 11 shows the largest value of the difference between the calcite volume fraction $\phi_{c}$ at $t=1$ day calculated with and without

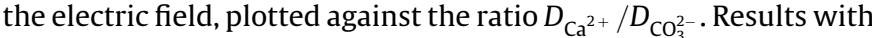
(dashed curve) and without flow (solid curve) are both shown. In the case without external flow, the largest difference of $\phi_{c}$ increases approximately from 0.13 to 0.18 (about a 38\% increase) as $D_{\mathrm{Ca}^{2+}} / D_{\mathrm{CO}_{3}^{2-}}$ decreases from 1 to 0.2 , while in the case with external flow, the largest difference of $\phi_{c}$ increases approximately from 0.075 to 0.10 (about a 33\% increase). Thus in both instances, larger differences in ion species diffusivities results in increased electric field acceleration. Also, acceleration is weakened when external flow is in presence.

To conclude, then, in this paper we apply a mixture model for biofilm-induced calcite precipitation through ureolysis to study selfinduced generation of an electric field due to different diffusivities of different ion species as well as the effects of the electric field on ionic transport and calcite precipitation. Our two-dimensional numerical simulations show that this electric field enforces charge neutrality, and with the parameter values used, it accelerates slowly diffusing ion species $\left(\right.$ e.g. $\mathrm{Ca}^{2+}$ ) into biomaterial, resulting in increased calcite precipitation rate. This observation holds either with or without external flow, but the effect of the electric field on calcite precipitation is weaker in the presence of external flow, presumably because diffusion is relatively less important.

The mechanism for enhanced precipitation appears to be as follows. (1) Ions with differently signed charges (in our case $\mathrm{CO}_{3}^{2-}$ and $\mathrm{Ca}^{2+}$ ) combine at a reaction site, thus creating a sink for these ion species and hence enhancing their gradients. (2) Resulting ion diffusion (with different diffusivities for the two ion species) induces an electric field which in turn speeds transport of the more slowly diffusing (and hence, reaction limiting) species. (The other ion species may actually suffer decreased diffusive transport, but since it is not limiting, does not slow the reaction rate.) (3) Enhanced transport of the limiting species results in an enhanced reaction rate. In the system studied here, enhanced reaction results in enhanced precipitation.

\section{Acknowledgments}

T. Zhang and I. Klapper are supported by NSF through Grant DMS-0934696. The authors thank Robin Gerlach and Philip Stewart for many helpful suggestions and comments.

\section{References}

[1] CRC Handbook of Chemistry and Physics, 88th ed., CRC Press, Taylor \& Francis Group, Boca Raton, FL, 2008.

[2] A. Ebigbo, R. Helmig, A.B. Cunningham, H. Class, R. Gerlach, Modelling biofilm growth in the presence of carbon dioxide and water flow in the subsurface, Adv. Water Resour. 33 (2010) 762-781.

[3] E. Alpkvist, I. Klapper, Description of mechanical response including detachment using a novel particle method of biofilm/flow interaction, Water Sci. Technol. 55 (2007) 265-273.

[4] S. Benini, C. Gessa, S. Ciurli, Bacillus pasteurii urease: a heteropolymeric enzyme with a binuclear nickel active site, Soil Biol. Biochem. 28 (1996) 819-821.

[5] M. Doi, Introduction to Polymer Physics, Oxford Science Publications, Oxford, 1995.

[6] H.J. Eberl, D.F. Parker, M.C.M. van Loosdrecht, A new deterministic spatiotemporal continuum model for biofilm development, J. Theor. Med. 3 (2001) 161-175.

[7] F.G. Ferris, V. Phoenix, Y. Fujita, R.W. Smith, Kinetics of calcite precipitation induced by ureolytic bacteria at 10 to $20^{\circ} \mathrm{C}$ in artificial groundwater, Geochim. Cosmochim. Acta 67 (2003) 1701-1722.

[8] Y. Fujita, F.G. Ferris, R.D. Lawson, F.S. Colwell, R.W. Smith, Calcium carbonate precipitation by ureolytic subsurface bacteria, Geomicrobiology 17 (2000) 305-318.

[9] J.L. Guermond, P. Minev, J. Shen, An overview of projection methods for incompressible flows, Comput. Methods Appl. Mech. Eng. 95 (2006) 6011-6045.

[10] H.S. Harned, B.B. Owens, The Physical Chemistry of Electrolytic Solutions, Reinhold Publishing Corporation, New York, NY, 1958.

[11] I. Klapper, J. Dockery, Mathematical description of microbial biofilms, SIAM Rev. 52 (2010) 221-265.

[12] L. Marini, Geological Sequestration of Carbon Dioxide: Thermodynamics, Kinetics and Reaction Path Modeling. Elsevier, 2007.

[13] A.C. Mitchell, F.G. Ferris, The coprecipitation of Sr into calcite precipitates induced by bacterial ureolysis in artificial groundwater: temperature and kinetic dependence, Geochim. Cosmochim. Acta (2005) 4199-4210.

[14] A.C. Mitchell, A.J. Phillips, M.A. Hamilton, R. Gerlach, K. Hollis, J.P. Kaszuba, A.B. Cunningham, Resilience of Bacillus mojavensis planktonic and biofilm cultures to supercritical $\mathrm{CO}_{2}$, J. Supercritical Fluids, doi:10.1016/j.supflu.2008. 07.005, 2008.

[15] C. Picioreanu, M.C.M. Loosdrecht, J.J. Heijnen, Mathematical modeling of biofilm structure with a hybrid differential-discrete cellular automaton approach, Biotech. Bioeng. 58 (1) (1998) 101-116.

[16] C. Picioreanua, I.M. Headc, K.P. Katurib, M.C.M. van Loosdrechta, K. Scott, A computational model for biofilm-based microbial fuel cells, Water Res. 41 (2007) 2921-2940.

[17] R.A. Robinson, R.H. Stokes, Electrolyte Solutions, Academic Press Inc, New York, 1959.

[18] Y. Saad, M.H. Schultz, GMRES: a generalized minimal residual algorithm for solving nonsymmetric linear systems, SIAM J. Sci. Statist. Comput. 7 (1986) 856-869.

[19] T. Shaw, M. Winston, C.J. Rupp, I. Klapper, P. Stoodley, Commonality of elastic relaxation times in biofilms, Phys. Rev. Lett. 93 (2004) 098102.

[20] L.A. Warren, P.A. Maurice, N. Parmar, F.G. Ferris, Microbially mediated calcium carbonate precipiration: implications for interpreting calcite precipitation 
and for solid-phase capture of inorganic contaminants, Geomicrobiol. J. 18 (2001) 93-115.

[21] Z. Xu, P. Meakin, Phase-field modeling of solute precipitation and dissolution, J. Chem. Phys. 129 (2008) 014705.

[22] T. Zhang, N. Cogan, Q. Wang, Phase-field models for biofilms. II. 2-D numerical simulations of biofilm-flow interaction, Commun. Comput. Phys. 4 (2008) 72-101.
[23] T. Zhang, I. Klapper, Mathematical model of biofilm induced calcite precipitation, Water Sci. Technol. 61 (2010) 2957-2964.

[24] Y. Zhang, R. Dawe, The kinetics of calcite precipitation from a high salinity water, Appl. Geochem. 13 (1998) 177-184.

[25] P. Zuddas, A. Mucci, Kinetics of calcite precipitation from seawater: II. The influence of the ionic strength, Geochim. Cosmochim. Acta 62 (1998) 757-766. 